\title{
46th Annual Meeting of the American Society of Clinical Oncology (ASCO)
}

Vom 4. bis 8. Juni 2010 fand in Chicago, Illinois/USA, die Jahrestagung der American Society of Clinical Oncology (ASCO) statt. Das Motto dieser Jahrestagung - "Advancing Quality through Innovation" - lässt bewusst verschiedene Gedankenverbindungen zu. Schließlich basieren die in den letzten Jahren errungenen Fortschritte in der Versorgung von Patienten mit Tumorerkrankungen auch auf kontinuierlichen Innovationen aus verschiedenen Bereichen wie beispielsweise der Forschung oder den Versorgungsstrukturen in der Onkologie. ASCO-Präsident Douglas
W. Blayney, Ann Arbor, Michigan/USA, wünscht sich eine entsprechende Weiterentwicklung für die Zukunft, um Patienten mit Tumorerkrankungen weiterhin bestmöglich versorgen zu können. Alle an der Versorgung von Tumorpatienten Beteiligten sieht Blayney dabei auch in der Verpflichtung, Innovationen unter Alltagsbedingungen, im Klinik- und Praxisalltag, umzusetzen und weiterzuentwickeln. Daher ist eine nach der ASCO-Jahrestagung fortgeführte intensive Diskussion der neu vorgestellten Innovationen wünschenswert.

\section{Lokal fortgeschrittenes oder metastasiertes Mammakarzinom}

\section{Eribulin-Monotherapie verlängert das Gesamtüberleben}

Ein medianes Gesamtüberleben von 13,12 Monaten mit Eribulin im Vergleich zu median 10,65 Monaten Gesamtüberleben mit einer Therapie nach Wahl des behandelnden Arztes, das ist das Ergebnis der Phase-III-Studie EMBRACE mit intensiv vorbehandelten Patientinnen mit lokal fortgeschrittenem oder metastasiertem Mammakarzinom (Twelves C et al., ASCO 2010: CRA1004^). Erstmals konnte in einer Phase-III-Studie mit einer Monotherapie in dieser Late-line-Therapiesituation ein Überlebensvorteil erreicht werden, so Chris Twelves, London/Großbritannien. Der Mikrotubuli-Inhibitor Eribulin ist ein synthetisches Analogon zu der aus dem marinen Schwamm Halichondria okadai isolierten zytotoxischen Substanz Halochondrin B. Der Wirkungsmechanismus des derzeit noch in der klinischen Entwicklung befindlichen Wirkstoffs unterscheidet sich von dem anderer Mikrotubuli-Inhibitoren wie z.B. den Taxanen.

In die EMBRACE-Studie wurden 762 Patientinnen mit lokal fortgeschrittenem oder metastasiertem Mammakarzinom eingeschlossen, die bereits 2 bis 5 Chemotherapien ( $\geq 2$ bei fortgeschrittener Erkrankung), einschließlich einer Vorbehandlung mit einem Anthrazyklin oder Taxan, erhalten hatten. Die Patientinnen wurden offen randomisiert im Verhältnis 2:1 entweder mit Eribulin (i.v. 1,4mg/ $\mathrm{m}^{2} \mathrm{KOF}$ an den Tagen 1 und 8 alle 21 Tage) behandelt oder bekamen eine Monotherapie nach Wahl des behandelnden Arztes (TPC=Treatment of Physician's Choice). Die Therapie in derTPCGruppe entsprach damit laut Twelves der im Klinikalltag, da eine Standardtherapie für Patientinnen in dieser Situation bislang nicht definiert ist. Der primäre Endpunkt der Studie war das Gesamtüberleben.

Zu Studienbeginn waren die Patientencharakteristika in den Behandlungsgruppen vergleichbar. In der TPC-Gruppe erhielten 96\% der Patientinnen eine Chemo- therapie, wobei Vinorelbin, gefolgt von Gemcitabin, Capecitabin und Taxanen, am häufigsten verwendet wurde. Eine Monotherapie mit Biologika oder ausschließlich Best Supportive Care wurde nicht eingesetzt.

\section{Vorteil zugunsten von Eribulin} Der primäre Endpunkt der Studie wurde erreicht, so Twelves: Beim Gesamtüberleben ergab sich ein signifikanter Vorteil zugunsten der Monotherapie mit Eribulin (Abb.).

Das Sicherheitsprofil der Behandlung mit Eribulin bezeichnete Twelves als handhabbar. Relevante Grad-3/4-Nebenwirkungen unter Eribulin waren $\mathrm{Neu}$ tropenie, Leukopenie, Asthenie/Fatigue und periphere Neuropathie.

In der Diskussion der Studienergebnisse bewertete Harold J. Burstein, Boston/USA,

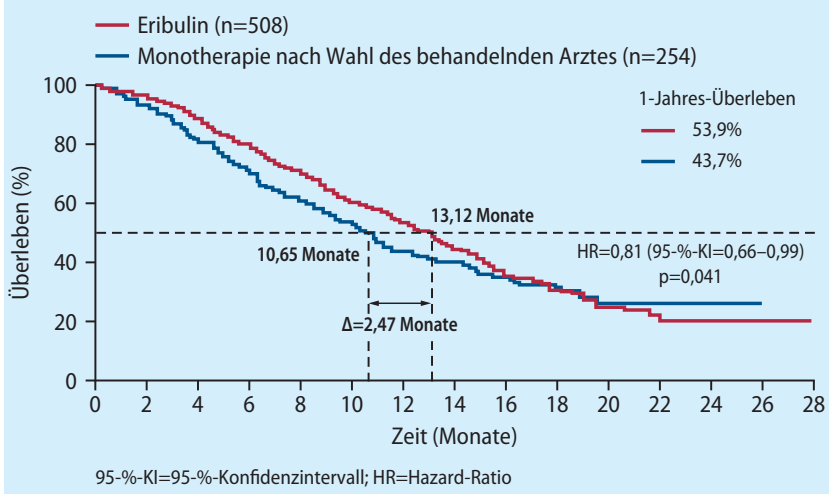

nach Twelves C et al., ASCO 2010: \#CRA1004^ and oral presentation

EMBRACE (Eisai Metastatic Breast Cancer Study Assessing Physician's Choice Versus Eribulin)-Studie - Gesamtüberleben

Eribulin als wichtige neue Behandlungsoption für intensiv vorbehandelte Mammakarzinom-Patientinnen. Wie die Substanz bestmöglich in bestehende Therapiealgorithmen integriert werden könne, müsse jedoch zunächst in weiteren Studien untersucht werden. $A M$ 